\title{
Mystique world of acrobatic molecular crystals
}

eljko Skoko ${ }^{1}$, Jasminka Popović ${ }^{2}$, Ivor Lončarić ${ }^{3}$

${ }^{1}$ Department Of Physics, Faculty Of Science, University Of Zagreb, Zagreb, Croatia, ${ }^{2}$ Institute Ruđer Bošković, Zagreb, Croatia, ${ }^{3}$ Centro de Física de Materiales CFM/MPC (CSIC-UPV/EHU), Donostia-San Sebastián, Spain

E-mail: zskoko@phy.hr

Everybody enjoys mystique and mystery that goes along with it, but they are hard to find outside the brother Grimm's fairy tales. But upon a travel to a nanoworld, not unlike Gulliver's voyage to Lilliput, in certain cases we can witness a truly fascinating and mystique phenomenon - a thermosalient effect. Thermosalient crystals, or more colloquially called jumping crystals, are intriguing materials that during heating/cooling exhibit joyful acrobatics in the form of hopping. These jumps are exhibited during the topotactic polymorphic phase transitions which are extremely fast and energetic so the crystals are balistically projected to heights several hundred times larger than their own dimensions. Thermosalient materials are also exhibiting huge technological potential because they are very promising candidates for fabrication of actuators on the microscopic level, such as nanoswitches, thermal sensors, artificial muscles, etc [1,2]. In the past several years, a large number of experimental studies was performed with the to unveil the origin of the thermosalient effect, but still today the real reason for this phenomenon remains foggy and mysterious. It is becoming obvious that the experimental methods solely are not enough to successfully tackle this challenge so it seems opportune to try to complement these results with the theoretical studies, employing the highly developed DFT calculations.

We present the results of such, to the best of our knowledge, first collaboration between experimental and theoretical studies performed on one of the thermosalient systems - $\mathrm{N}^{\prime}$-2-propylidene-4-hydroxybenzohydrazide. This system experiences even three polymorphic thermosalient transition, one of them being irreversible and the other two reversible. As with the large majority of thermosalient compounds, it is also characterised with anisotropic thermal expansion. It shows immense negative uniaxial thermal expansion (along b direction), which we suspect is the governing force for the thermosalient phenomenon in this system. Our first principle electronic structure calculations prove that it is a direct consequence of the negative uniaxial compressibility. Elastic properties are also shown to be the origin of the reversibility or irreversibility of the thermosalient phase transitions. And finally, our DFT calculations suggest that excitations of the lowenergy phonons provide a necessary trigger for the thermosalient effect in $\mathrm{N}^{\prime}$-2-propylidene-4-hydroxybenzohydrazide, propelling the system over the energy barrier between the thermosalient phases.

[1] Skoko, Ž. et al. (2010). J. Am. Chem. Soc. 132, 14191-14202.

[2] Nath, N. K. et al. (2014). CrystEngComm 2014, 1850-1858.

a)

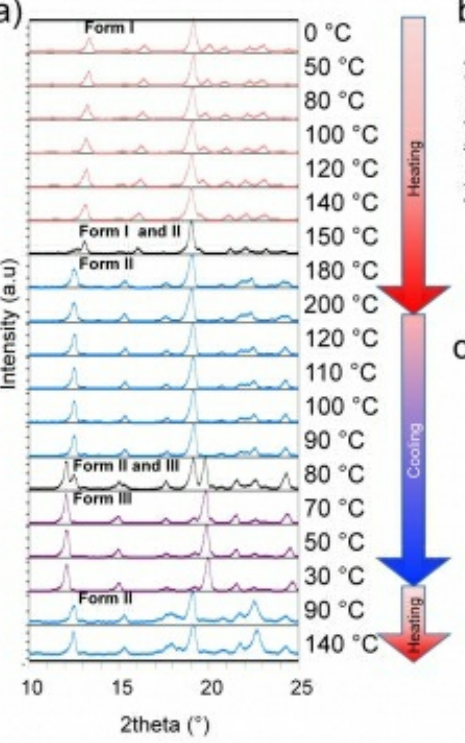

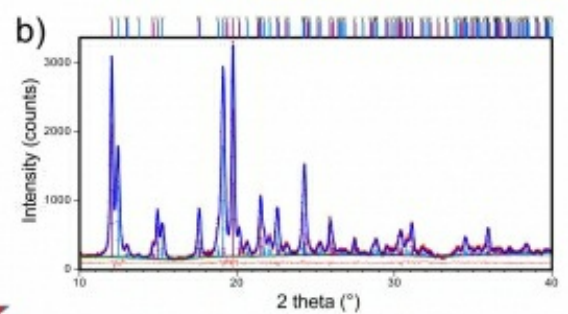

Compression in $b$ direction

c)

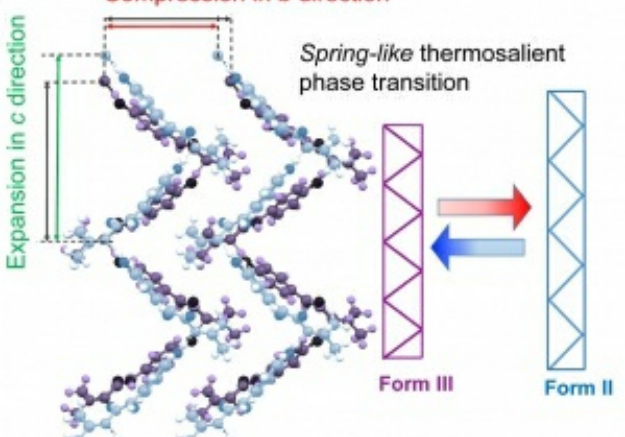

Keywords: thermosalient effect, phase transition, DFT 